\title{
ANN System Identification for Rapid Battery Charger using Parallel 3 Parent Genetic Algorithm
}

\author{
Ashima Kalra, Shakti Kumar, Sukhbir Singh Walia
}

\begin{abstract}
Model identification is one of the main concerns in the field of system modeling. The complete modeling of an ANN system using input output data consists of two processes: architecture selection in which number of hidden layers and the number of neurons in each hidden layer is to be decided. This is then followed by training of system by the given training data. The problem here is formulated as search and minimization problem. This paper presents the identification of ANN system for rapid Nickel Cadmium (Ni-Cd) batteries charger by applying a new version of Genetic algorithm called as parallel 3 parent genetic algorithm (P3PGA). It is a multi population based 3 parent genetic algorithm (3PGA) .The proposed approach was implemented using MATLAB R2018A and was observed to be computationally more efficient with minimum MSE. With increase in number of iterations, system performance gets improved. We further compared results of the proposed algorithm with the results of other recent soft computing based algorithms as well classical learning based algorithms namely, big bang big crunch (BB-BC), parallel big bang big crunch (PBB-BC), Levenberg-Marquardt algorithm (LM), error back propagation (EBP), Resilent prop (RPROP), particle swarm optimization (PSO), ant colony optimization (ACO) and artificial bee colony $(A B C)$ for $A N N$ model identification. The proposed algorithm outperformed all of the other 8 algorithms.
\end{abstract}

Index Terms: model identification, 3 parent genetic algorithm (3PGA), parallel 3 parent genetic algorithm (P3PGA)

\section{INTRODUCTION}

Neural network is an information processing paradigm inspired by the structure of brain. Likewise brain, it also consists of a number of small units called as neurons that are capable of doing the computation in parallel. It is most often modeled as an optimization process wherein the error is minimized as the learning takes place. This paper discusses ANN system design for rapid Nickel Cadmium (Ni-Cd) battery charger from the given training data set. The complete modeling of an ANN system consists of two processes: first the selection of ANN architecture in which the number of hidden layers and the number of neurons in each hidden layer is to be decided. Second is the training of this ANN system by the given training data. The problem here is formulated as a search and minimization problem.

Revised Manuscript Received on June 15, 2019.

Ashima Kalra, PhD research scholar, Punjab technical university,Punjab, India.

Prof. (Dr.) Shakti Kumar, Director, Panipat Institute of engineering Technology, Panipat, Haryana, India

Dr. Sukhbir Singh Walia , Registrar, Punjab technical university, Punjab, India.
The optimization algorithms are applied in a way to automatically adjust the number of hidden layers, neurons in each of the hidden layers and identified values of synaptic weights in such a way so as to minimize the objective function i.e. MSE.

$$
M S E=\frac{1}{N} \sum_{k=1}^{N}[O A-O C]^{2}
$$

where OA is actual output of the system and $\mathrm{OC}$ is the calculated output of the system.

Back propagation is the most traditional gradient-based method to train an artificial neural network and which was first introduced by Werbos [1]. It is used to adjust the network's weights and biases by calculating the gradient of the error. Usually, back propagation adjusts the weights of neural network through some random weight initialization. However, training the neural networks with random initial weights may cause two main drawbacks: trapping into local minima and converging slowly [2], [3]. Due to the limitations of back-propagation neural networks, various global search techniques (e.g., genetic algorithm and particle swarm optimization, ant colony optimization) have been presented to overcome these shortcomings [4]-[6]. These are the techniques for optimization and learning.

This paper consists of $\mathrm{V}$ sections. Section II gives the literature review of genetic algorithm followed by the work done related to three parent GA. Section III discusses the ANN modeling process and 3 parent GA based ANN system for rapid battery charger. Section VI discusses the simulation, observations, results and comparison of the system identification by proposed approach. Section V concludes the paper.

\section{LITRATURE REVIEW}

\section{A. Related Work}

Genetic algorithms (GAs) are a global optimization process used for ANN learning or training. GA is used to evolve the architecture of neural network and the connection weights as well. It is expected to avoid local optima frequently by promoting exploration of the search space, in opposition to the exploitative trend usually allocated to local search algorithms like BP or LM. David J. Montana [6] proposed NN weight selection using GA which was implemented with feed 
forward sigmoidal neural networks. It does not use any encoding scheme for the networks but rather uses the networks themselves as the chromosomes. Montana and L. Davis [7] have explained that multilayered feed forward neural networks possess a number of properties which make them particularly suited to complex pattern classification problem. Along with they also explained the concept of genetics and neural networks. Alba et al [8] proposed training Neural Networks with GA Hybrid Algorithms. They suggested the concept of weak hybridization (just the combination of two algorithms) by introducing and testing GA with the BP algorithm (GABP), and a GA with LM (GALM). In both cases the problem-specific algorithm (BP and $\mathrm{LM}$ ) is used as a mutation-like operation of the general search template (GA). H Kitanao [9] compared genetic algorithms (GA) with BP (back propagation) and presented a hybrid approach GA-BP which was proved faster than GA alone. GA was found to be equally efficient to the faster variants of BP in small scale networks but found less efficient in large networks. J.N.D Gupta et.al [10], Zhen Guo Che et.al [11] compared Standard EBP with GA for optimizing artificial neural networks. GA was found to be better than EBP in effectiveness, ease-of-use and efficiency in training NNs. V. Saishanmuga Raja et al in [12] compared three optimization techniques GA, ACO and PSO in the biomedical application based on processing time, accuracy and time taken to train Neural Networks. The author concluded that GA outperformed the other two algorithmsACO and PSO and is most suitable for training the neural network with minimum time and minimum mean square error. Richa Mahajan et al [13] used the concept of combining neural networks with genetic algorithm. The author proposed a genetic algorithm implementation in order to give a maximal approximation of the problem with the reduction of cost.

\section{B. Parallel 3 Parent Genetic algorithm (P3PGA)}

The above mentioned genetic algorithm is a two-parent algorithm including the process of selection, crossover and mutation. There are cases where offspring has to suffer due to the bad characteristics of one parent. Mitochondrial diseases are caused by the faulty mitochondria inherited from the parents So as to overcome this, multiparent recombination is found to a good solution for such cases as suggested by the literature [14], [15]. A E Eiben et al [16] investigated the genetic algorithm where the effect of more than two parents is involved in the recombination process. The author introduced the two multiparent recombination schemes i.e gene scanning and diagonal crossover for the problem of numerical optimization and constrained optimization. It was found that in some cases 2 parents GA is optimal while in some cases, a greater number of parents gave better results. In one of such techniques called spindle nuclear transfer [17], Dr. Zang Johan at his fertility Centre in New York City removed the nucleus from the defective egg cell of mother and inserted it into the cell body of a donor whose nucleus was removed. The donor's cell body had healthy mitochondria. The resulting egg cell with the nucleus DNA of the mother and the healthy mitochondrial DNA from the donor was fertilized with the father's sperm. This lead to the birth of the first 3 parent-child with healthy mitochondria on 6 April 2016. Based on the above process Amar et al. [18] proposed 3PGA global optimization algorithm and proved it to be much superior to 16 other optimization algorithms. Further, a new version called parallel 3 parent GA is proposed by Amar et al. [19] for automatic test case generation. These techniques are further used by many researchers for energy efficient routing in wireless sensor networks [20].

\section{ANN BASED SYSTEM IDENTIFICATION USING P3PGA}

In literature, many authors [21], [22] presented identification of Fuzzy model for the rapid Nickel-Cadmium (Ni-Cd) battery charger using soft computing approaches. Many soft computing approaches have also been applied for ANN model identification in literature [23], [24].

In this paper, we have applied P3PGA for identification of ANN model for rapid Ni-Cd battery charger to evolve the architecture as well as for training purpose. The main objective is to charge the batteries as quickly as possible but without doing any damage to them. The two input variables to control charging Rate $(\mathrm{Ct})$ are absolute temperature $(\mathrm{T})$ and its Temperature gradient. The model belongs to the control system category as shown in figure 1 .

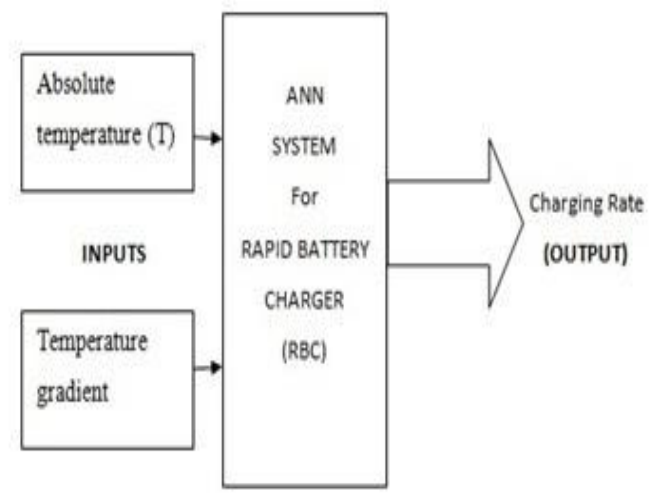

Fig. 1 Block Diagram of the ANN System for RBC

It is a two input and single output control problem. Modeling an ANN System is a complex process involving a number of steps. This complexity further increases with the increase in the number of input parameters and the number of hidden layers. The main steps to be followed for designing a complete ANN System are given as below.

1. Begin zero number of hidden layers i. e $\mathrm{NH}=0$

2. Fix the number of neurons in each hidden layer.(values of $\mathrm{k} 1, \mathrm{k} 2 \ldots$ and so on)

3. Randomly initialize the weights of ANN

4. For each training pattern, evaluate output and error between the computed and desired output.

5. Compute mean square error for the model (MSE).

6. Minimize the objective function (MSE) by adjusting the weights using the proposed approach i.e P3PGA

7. If MSE is acceptable or termination criterion is met,

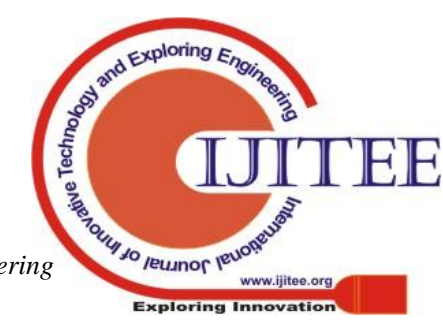


then go to step 9, else if the number of hidden layers is non zero then increase the number of neurons in the hidden layers. After an upper limit of the number of neurons in the hidden layers has reached and if the performance is still not acceptable we increase the number of hidden layers.

8. Go to step 4.

9. Stop

For identifying the ANN rapid battery charger model using the above-mentioned optimization algorithm, a population of individuals is randomly generated. Each individual in the population represents a complete artificial neural-network system. The size of an individual i.e. set of synaptic weights is variable and depends upon factors like the number of inputs, number of hidden layers, number of neurons in each hidden layer.P3PGA is multi population algorithm based on three parent GA (3PGA) [18]. The pseudo code of P3PGA is given below.

\section{Start}

Generate $\mathrm{n}$ populations randomly by chromosomes

For Gen $=1$ : Number of Generations

For $\mathrm{i}=1: \mathrm{N}$

Make mitochondrial change to ith population to generate 3Parent (3-P) population.

$/ /^{*}$ we implement this process by adding a small random number in every gene of the individuals. *//

Add current ith two parent (2-P) population with new 3-P population.

Calculate fitness, sort population and select best fittest individuals.

Find and Record fittest solution.

Produce new ith 2-p population by applying general Genetic Algorithm:-

1) Initialize the population randomly by chromosomes.

2) Evaluate the Fitness and compute the elite.

3) If it satisfies the fitness function than stop otherwise go to the next step.

4) Generate the new population around the elite by adding or subtracting a small number in it.

5) Repeat step four.

Check bounds violation \& correct if needed.

Select local best candidates lbest(i) for ith population;

End for

From amongst the local $\mathrm{N}$ best candidates select the globally best gbest candidate;

For $\mathrm{I}=1$ : $\mathrm{N}$ do $/ / *$ move local best towards global best

With a given probability replace a gene of lbest(i) with the corresponding gene of global best (gbest(i) ) candidate;

End for

End for

End

\section{SIMULATION RESULTS}

The suggested approach has been applied for identification of ANN model for the rapid Nickel-Cadmium (Ni-Cd) battery charger [25]. The objective of this charger was to charge 2AA Ni-Cd batteries as quickly as possible but without doing any damage to them. Input-output data consists of 561 points available at www.cilab.in. For this charger, the two input variables used to control the charging rate $(\mathrm{Ct})$ are absolute temperature of the batteries $(\mathrm{T})$ and its temperature gradient $(\mathrm{dT} / \mathrm{dt})$.

In order to validate the proposed approach, we have implemented the proposed approach of ANN model identification in MATLAB R2018a. We have used a rapid battery charger (RBC) data consisting of 561 patterns to evolve the architecture as well as for training purpose. With the increase in the number of layers or the number of neurons in these layers the number of weights i.e. the size of individual increases. We have used $20 \%$ of the data for training purposes. For each of the implemented approach, 15 trials were taken with 500, 1000, 2000, 5000 and 10,000 iterations and the MSE of each evolved model is then recorded. Table I shows the different parameters considered for the ANN model identification for the rapid battery charger system with two inputs and one output.

TABLE I: Parameters for ANN model identification

\begin{tabular}{|l|l|}
\hline Parameters & Values for RBC \\
\hline Size of population & 100 \\
\hline Number of hidden layers & 2 \\
\hline Number of neuron in the first hidden layer & 5 \\
\hline Number of neuron in the first hidden layer & 3 \\
\hline Number of iterations & $500,1000,20005000,10,000$ \\
\hline Number of input variables & 2 \\
\hline
\end{tabular}

TABLE II: Performance comparison of proposed approach P3PGA with 3PGA

\begin{tabular}{|c|c|c|}
\hline Iterations & $\begin{array}{c}\text { 3parentGA } \\
\text { (3PGA) }\end{array}$ & $\begin{array}{c}\text { Parallel3PGA } \\
\text { (P3PGA) }\end{array}$ \\
\hline 500 & 0.0945 & $7.89 \mathrm{E}-04$ \\
\hline 1000 & 0.0175 & $7.53 \mathrm{E}-04$ \\
\hline 2000 & 0.0263 & $7.49 \mathrm{E}-04$ \\
\hline 5000 & 0.0163 & $7.47 \mathrm{E}-04$ \\
\hline 10000 & 0.025 & $7.57 \mathrm{E}-04$ \\
\hline
\end{tabular}

Table II compares the performance of the proposed P3PGA with simple three parent GA. MSE is found to be the minimum in the case of P3PGA than that of simple three parent GA (3PGA) even with the increase of iterations also in all the cases.

Simulation results show that the ANN model for 500 iterations leads to MSE of 
7.89e-0.4, however on increasing the iterations; system performance gets improved, as for 1000 iterations.MSE of the model gets reduced to 7.53 e-04. Figure 2 represent the simulation graph depicting the improvement in the system with an increase in the number of iterations for 3PGA. In this, the value of performance parameter i.e MSE is plotted for different values of iterations i.e 500, 1000, 2000 and 10000. MSE decreases with the increase of iterations

Figure 3 represents the simulation graph depicting the improvement in the system with an increase in the number of iterations for 3PGA. In this, the value of performance parameter i.e. MSE is plotted for different values of iterations i.e. 500, 1000, 2000 and 10000.MSE value decreases with the increase of iterations till 5000 iterations. When the iterations is increased from 5000 to 10000 , MSE is found to be slightly increased. Still MSE of P3PGA is found to be the minimum out of two approaches

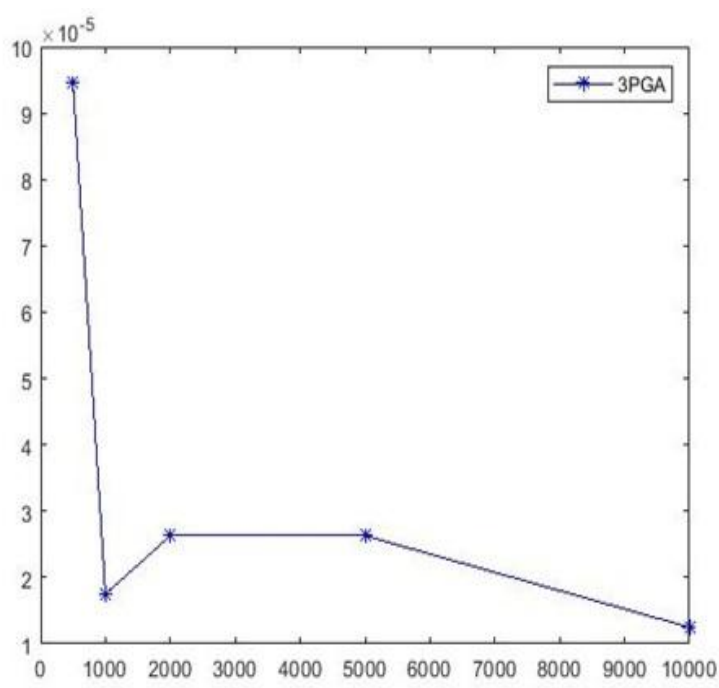

Fig 2. MSE Vs Iterations for 3PGA

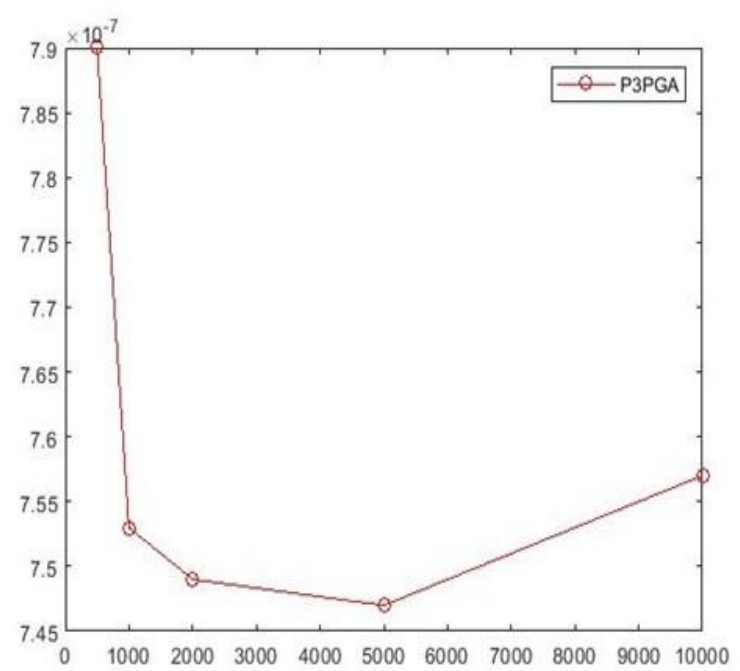

Fig. 3 MSE Vs Iterations for P3PGA

Table III compares the performance of proposed algorithm with other approaches namely error back propagation (EBP), Levenberg-Marquardt algorithm(LM), Resilient prop(RPROP), particle swarm optimization (PSO), ant colony optimization(ACO) and artificial bee colony(ABC) and big bang big crunch (BBBC) and parallel big bang big crunch (PBBBC) based model identification approaches . The value of MSE with this proposed approach has been found to be far superior to the other 8 algorithms. It clearly highlights the performance improvement provided by the proposed algorithm. MSE comes out to be minimum in the case of parallel 3 parent GA as compared to other approaches. With the increase in the number of iterations, MSE remains minimum out of all approaches.

\section{CONCLUSION}

This paper presented a new approach to the ANN model identification $\mathrm{Ni}-\mathrm{Cd}$ rapid battery charger. ANN model identification deals with evolving optimal ANN architecture wherein we select the optimal number of layers and optimal numbers of neurons in each hidden layer, for a given type of activation function for the neurons. The proposed algorithms simultaneously train the ANN for the optimal value of synaptic weights so as to minimize MSE. We evolved a rapid battery charger (RBC) from the given training data which is a control system problem. The proposed approaches evolved the architecture as well as trained the ANN simultaneously to produce the optimal models. We used only about $20 \%$ of the data for training purposes.

TABLE III: Performance comparison of proposed approachP3PGA with different approaches

\begin{tabular}{|c|c|c|c|c|c|c|c|c|c|c|c|}
\hline \multirow{11}{*}{$\begin{array}{l}\text { Perform } \\
\text { ance } \\
\text { measure } \\
\text { (MSE) }\end{array}$} & \multicolumn{11}{|c|}{ Model Identification Approach } \\
\hline & & PSO & $\begin{array}{l}\text { BBB } \\
C\end{array}$ & $A C O$ & EBP & $\begin{array}{l}\text { RPR } \\
\text { OP }\end{array}$ & LM & $A B C$ & $\begin{array}{l}\text { PBB } \\
\text { BC }\end{array}$ & $\begin{array}{l}3 P G \\
A\end{array}$ & $\begin{array}{l}P 3 P \\
\text { GA }\end{array}$ \\
\hline & $\begin{array}{l}\text { iterations= } \\
500\end{array}$ & $\begin{array}{l}0.02 \\
17\end{array}$ & $\begin{array}{l}0.00 \\
53\end{array}$ & $\begin{array}{l}0.01 \\
57\end{array}$ & $\begin{array}{l}0.11 \\
74\end{array}$ & $\begin{array}{l}0.11 \\
71\end{array}$ & $\begin{array}{l}0.19 \\
66\end{array}$ & $\begin{array}{l}0.11 \\
43\end{array}$ & $\begin{array}{l}0.00 \\
18\end{array}$ & $\begin{array}{l}0.09 \\
45\end{array}$ & $\begin{array}{l}7.89 \\
\text { E-04 }\end{array}$ \\
\hline & iterations $=$ & 0.03 & 0.00 & 0.01 & 0.11 & 0.11 & 0.19 & 0.11 & 1.39 & 0.01 & 7.53 \\
\hline & 1000 & 9 & 53 & 14 & 78 & 7 & 66 & 2 & E-05 & 75 & $E-04$ \\
\hline & iterations= & 0.01 & 0.00 & 0.01 & 0.11 & 0.11 & 0.19 & 0.11 & 8.68 & 0.02 & 7.49 \\
\hline & 2000 & 65 & 53 & 42 & 76 & 7 & 66 & 43 & E-06 & 63 & $E-04$ \\
\hline & iterations= & 0.00 & 0.01 & 0.01 & 0.11 & 0.11 & 0.19 & 0.11 & 6.10 & 0.01 & 7.47 \\
\hline & 5000 & 61 & 23 & 32 & 77 & 69 & 66 & 13 & E-05 & 63 & E-04 \\
\hline & iterations= & 0.01 & 0.00 & 0.01 & 0.11 & 0.11 & 0.19 & 0.11 & 0.00 & 0.02 & 7.57 \\
\hline & 10000 & 99 & 53 & 27 & 73 & 69 & 66 & 79 & 19 & 5 & E-04 \\
\hline
\end{tabular}

For each of the proposed algorithm, we conducted 15 trials for recording MSE. We evolved the ANN models and recorded the MSE of each evolved model. The MSE values of the evolved system for all the trials were recorded. We observed that simple 3parent GA evolved the ANN RBC with minimum MSE of 0.0175 whereas the Parallel 3 parent GA(P3PGA) approach evolved the RBC model with minimum MSE of $7.53 \mathrm{e}-04$ in 1000 iterations. Thus we conclude that, based on the MSE performance parameter, the parallel 3 parent GA (P3PGA) approach performs better than the simple 3 parent GA based approach(3PGA) and results in minimum MSE as 
compared to other approaches and remained minimum with the increase of iterations as well.

\section{ACKNOWLEDGMENT}

I would like to thank Dr. (Prof.) Shakti Kumar, Director, PIET, Panipat and Dr Sukhbir Singh Walia, Registrar, IK Gujral Punjab Technical University, for their invaluable guidance and sincere support for this work.

\section{REFERENCES}

1. Werbos, P.J., "The Roots of Backpropagation", John Wiley k Sons, Inc., New York, 1994.

2. Sehgal et.al, "Minimization of Error in Training a Neural Network Using Gradient Descent Method", International Journal of Technical Research(IJTR), Vol 1, Issue 1, Mar-Apr 2012, pp 10-12.

3. Ahmad Hashim Hussein Aal-Yhia, and Ahmad Sharieh, "An Energy Backpropagation Algorithm", Proceedings of the World Congress on Engineering WCE 2007, Vol I, July 2007,

4. Byoung Tak Zhang et.al., "Evolving Optimal Neural Networks Using Genetic Algorithms with Occam_s Razor", Complex Systems,7(3),199-220,1993

5. L. Yan-Peng, W. Ming-Guang, and Q. Ji-Xin, "Evolving neural networks using the hybrid of ant colony optimization and bp algorithm," in Advances in Neural Networks - 3rd International Symposium on Neural Networks, ser. LNCS, vol. 3971. Springer-Verlag, 2006, pp. 714-722.

6. David J. Montana, "Neural Network Weight Selection Using Genetic Algorithms", pp 1-17.

7. David J. Montana and Lawrence Davis, "Training Feedforward Neural Networks Using Genetic Algorithms", Machine Learning, pp 762-767.

8. Alba E., Chicano J.F. (2004) Training Neural Networks with GA Hybrid Algorithms. In: Deb K. (eds) Genetic and Evolutionary Computation - GECCO 2004. GECCO 2004. Lecture Notes in Computer Science, vol 3102. pp 852-863 Springer, Berlin, Heidelberg

9. Hiroaki Kitano, "Empirical Studies on the Speed of Convergence of Neural Network Training using Genetic Algorithms", Machine Learning, AAAI-90 Proceedings, pp 789-795.

10. Jatinder N.D. Gupta, Randall S. Sexton, "Comparing backpropagation with a genetic algorithm for neural network training”, Elsevier, Omega 27 (1999), pp 679-684.

11. Zhen-Guo Che, Tzu-An Chiang, and Zhen-Hua Che, "Feed forward Neural Networks Training: A Comparison Between Genetic Algorithm and Back-propagation Learning Algorithm", International Journal of Innovative Computing, Information and Control, ICIC International 2011 ISSN 1349-4198, Volume 7, Number 10, October 2011, pp. 5839 5850.

12. V. Saishanmuga Raja and S.P. Rajagopalan, "A Comparative Analysis of Optimization Techniques for Artificial Neural Network in Bio-Medical Applications", Journal of Computer Science 10 (1): pp 106-114, Science Publications, 2014

13. Richa Mahajan, Gaganpreet Kaur, Guru Nanak Dev University, Amritsar.Neural Networks using Genetic Algorithms, International Journal of Computer Applications (0975 - 8887), Volume 77- No.14, September 2013

14. Effect of the number of parents on the performance of multi-parent genetic algorithm, November 2016 , Conference: 2016 11th International Conference on Knowledge, Information and Creativity Support Systems (KICSS), Seng Pan That Pann Phyu, Gun Srijuntongsiri

15. Shih-Hsin Chen, Min-Chih Chenb, Pei-Chann Chang, V. Mani, Multiple parents crossover operators: A new approach removes the overlapping solutions for sequencing problems, Applied Mathematical Modelling, Volume 37, Issue 5, March 2013, 2737-2746.

16. Eiben A.E., Raué P.E., Ruttkay Z. (1994) Genetic algorithms with multi-parent recombination. In: Davidor
Y., Schwefel HP., Männer R. (eds) Parallel Problem Solving from Nature - PPSN III. PPSN 1994. Lecture Notes in Computer Science, vol 866. Springer, Berlin, Heidelberg

17. Paula Amato, Masahito Tachibana, Michelle Sparman, and Shoukhrat Mitalipov, "Three-Parent IVF: Gene Replacement for the Prevention of Inherited Mitochondrial Diseases", Fertility and Sterility, 101(1), 2014, pp. 31-35.

18. A Singh, S Kumar, A Singh, SS Walia , "Three-parent GA: A Global Optimization Algorithm", Journal of Multiple-Valued Logic \& Soft Computing, Vol. 32, ,2019 ,pp 407-423.

19. Nargis Akhter, Amar Singh, Mr. Guljar Singh, Automatic Test Case Generation by using Parallel 3 Parent Genetic Algorithm, International Journal for Research in Applied Science \& Engineering Technology (IJRASET), Volume 6 Issue VII, July 2018

20. A Singh, Shakti Kumar, Sukhbir Singh Walia, P3PGA: Multi-Population 3 Parent Genetic Algorithm and its Application to Routing in WMNs, International Journal of Advanced Research in Computer Science, Volume 8, No. 5, May - June 2017.

21. Ankita et al, Fuzzy system identification for Ni-Cd battery charger using genetic algorithm, International Journal of Engineering Research \& Technology (IJERT), Vol. 1, Issue 5, July -2012

22. S Kumar, P Kaur and A Singh," Fuzzy Model Identification: A Firefly Optimization Approach. International Journal of Computer Applications 58(6):1-8, November 2012.

23. A Kalra, S Kumar, S S Walia, "ANN Model identification: Two Soft Computing Based Approaches", International Journal of Research and Analytical Reviews, Vol. 4, issue 2, June 2017, pp 79-86.

24. A Kalra, S Kumar, S S Walia, ANN Model Identification: A BB-BC Optimization Algorithm Based Approach, International Journal of Computer Sciences and Engineering, Vol.-6, Issue-12, Dec 2018, pp 264-271.

25. A Khosla, S Kumar, and K K Aggarwal,2002. Design and development of RFC-10: A fuzzy logic based rapid battery charger for Nickel-Cadmium batteries. HiPC (High Performance Computing), Workshop on Soft Computing, Bangalore, 9-14.

\section{AUTHORS PROFILE}

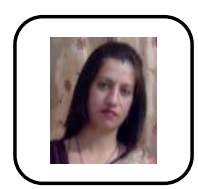

Ashima Kalra , Gold Medalist in B Tech in Electronics from Kurukshetra University, Kurukshetra in 2003 .Received M.Tech degree from Punjab Technical University, Kapurthla (Punjab)in 2008 and pursuing PhD from Punjab Technical University, Kapurthla (Punjab )in the field of soft Computing. She has published more than 25 papers in reputed journals and 3 book chapters in Springer series. Her research activities include designing model identification using neural networks, fuzzy systems, supervised learning .machine learning. Lifetime IEEE member and has been serving as a fellow member of IEEE Delhi section, India. Organized 3 IEEE international conferences as a chairperson.

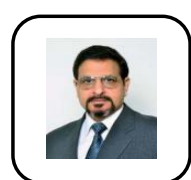

Prof. ( Dr.) Shakti Kumar did his MS ( Electronics \& Control) from BITS, Pilani, India and Ph.D. (Electronics \& Computer Engg.) from National Institute of Technology, Kurukshetra, India. Presently, he is Director, Panipat Institute of engineering Technology, Panipat, Haryana, India. His research areas include Communication Networks, Soft Computing and Intelligent Systems. He has published more than 200 research articles in various Journals and Conferences and 7 book chapters. He has organized 7 international and 6 national conferences in the areas of intelligent systems and networks. 
ANN System Identification for Rapid Battery Charger using Parallel 3 Parent Genetic Algorithm

Dr. Sukhbir Singh Walia has done his B.Tech. from Guru Nanak Dev University Amritsar, M.Tech \& Ph.D. from Punjab Technical University (PTU), Kapurthla (Punjab)

India. His research areas include Soft Computing,

Education Management, Mobile Communication, and

Wireless Sensor Networks. He has published around 28

research articles in various Journals and Conferences. Currently he is Director, International Resource Centre in Human Values and Professional Ethics at PTU. 